\section{Spontanverschluss einer zerebralen arteriovenösen Malformation durch Thrombose des Nidus und der drainierenden Venen}

Unter den intrazerebralen Gefäßmissbildungen haben arteriovenöse Malformationen (AVM) nach großen Autopsieserien eine Inzidenz von $0,04 \%$ bis $0,52 \%$. In den USA werden jährlich ca. 10 zerebrale AVM pro 1 Million Einwohner diagnostiziert. In der Regel erfolgt deren Behandlung operativ, endovaskulär (Embolisation) oder radiochirurgisch (Berlis A. et al.; Akt. Neurologie 1999, 26:270). Spontanverschlüsse von zerebralen arteriovenösen Malformationen sind selten und bezüglich ihres Pathomechanismus oder möglicher prädisponierender Faktoren weitgehend unverstanden. Wir berichten über einen Patienten mit angiographisch und kernspintomographisch dokumentiertem Spontanverschluss einer links parietookzipitalen pialen AVM durch frische Thrombose des Angiomnidus und der drainierenden Venen.

\section{Fallbericht}

Bei einem 61-jährigen Patienten traten im Sommer 2000 Sprachstörungen im Sinne einer motorischen Aphasie auf, die sich im September 2000 wiederholten, so dass zur Abklärung ein MRT des Schädels durchgeführt wurde. Hierbei zeigte sich eine links parieto-okzipitale AVM, die angiographisch als piale AVM weiter differenziert werden konnte, die von zwei Feedern aus dem linken erweiterten parietalen Mediahauptast gespeist wurde (Abb. 1). Der Nidus wies einen Durchmesser von $2 \mathrm{~cm}$ auf. Die venöse Drainage erfolgte über zwei oberflächliche Venen. Im weiteren Verlauf kam es zu mehreren fokalen Anfällen mit absenceartigen $\mathrm{Zu}$ ständen und einem Grand-Mal-Anfall.

4 Monate später stellte sich der Patient zur Behandlung in unserer Klinik vor. Klinisch wies der Patient kein neurologisches Defizit auf, berichtete allerdings über einen weiteren unmittelbar am Wochenende zuvor stattgehabten GrandMal-Anfall. Die Angiographie erfolgte in Interventionsbereitschaft und zeigte auch bei selektiver Darstellung des linken parietalen Mediaastes eine spontan verschlossene AVM bei weiterhin erweiter-

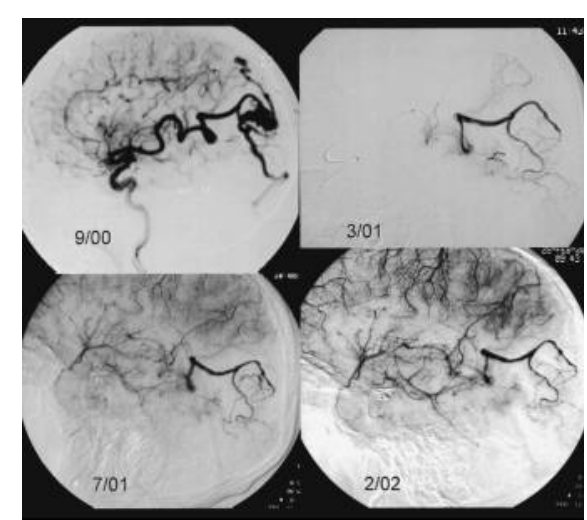

Abb. 1 Angiographie der ACl links im zeitlichen Verlauf über 16 Monate mit Spontanverschluss einer links parieto-okzipitalen AVM.

tem Lumen der zuführenden Arterie sowie verschlossene ehemals drainierende Venen (Abb.1). Bei Aneurysmaverdacht erfolgte eine Rotationsangiographie der linken ACImit $13 \mathrm{ml}$ Kontrastmittel bei einem Flow von 2,5 ml/s. Hierbei waren zwei Aneurysmen am Abgang der A. communicans posterior sowie flussassoziiert am proximalen parietalen Mediahauptast nachweisbar (Abb. 2). Computertomographisch konnten im Bereich des Nidus eine schollige Verkalkung sowie eine keilförmige auf das Parenchym beschränkte Dichteminderung festgestellt werden. MR-tomographisch erhärtete sich der Verdacht auf einen frischeren venösen Infarkt mit Signalanhebung in der diffusionsgewichteten und $\mathrm{T}_{2}$-gewichteten Sequenz sowie eine frische Thrombose der ehemals drainierenden Venen und des Nidus (Abb. 3).

4 Monate nach unmittelbar angeschlossener Aneurysmaoperation erfolgten eine Kontrollangiographie (Abb.1) und ein MRT (Abb.3). Weiterhin war keine AVM abgrenzbar. Beide Aneurysmen waren komplett ausgeschaltet. Es zeigte sich ein deutlich verlangsamter Fluss im erweiterten parietalen Mediaast. Die angiographisch weiterhin verschlossenen ehemals drainierenden Venen wiesen im MRT alte thrombotische Veränderungen auf. In der zweiten angiographischen Kontrolle nach einem Jahr wies der parietale Mediaast neben der Flussverlangsamung und nahe-

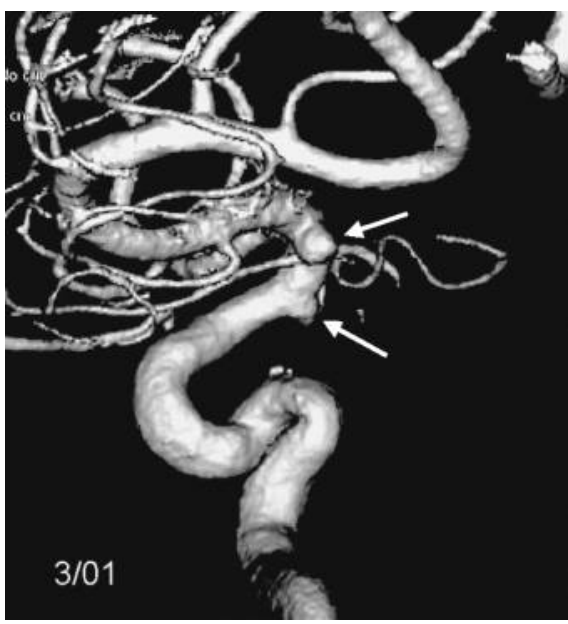

Abb. 2 Rotationsangiographie der linken $\mathrm{ACl}$ mit 3D-Rekonstruktion und Nachweis eines flussassoziierten proximalen Aneurysmas des angiomversorgenden parietalen Mediahauptastes sowie eines weiteren Aneurysmas am Abgang der A. communicans posterior.
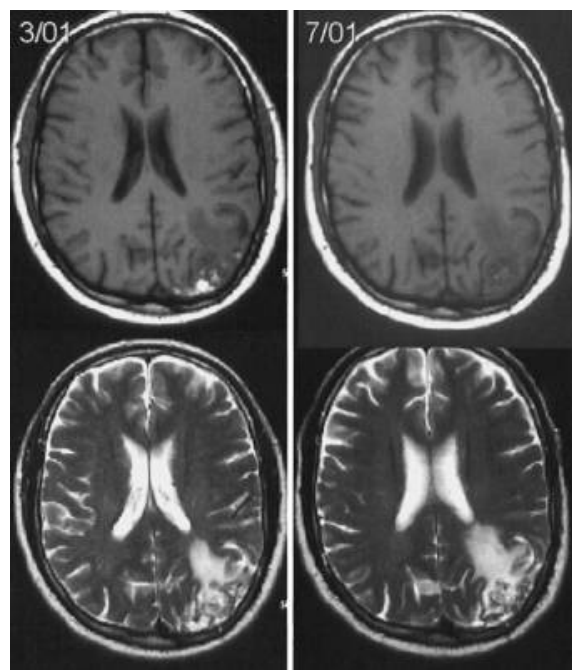

Abb. 3 MRT mit $\mathrm{T}_{1}$ - und $\mathrm{T}_{2}$-gewichteten $\mathrm{Se}$ quenzen mit frischer Thrombose einer drainierenden Vene sowie des Nidus (3/01). MRT-Verlaufskontrolle nach 4 Monaten (7) 01) mit überwiegend isointenser Darstellung des thrombotisch verschlossenen ehemaligen Nidus und der drainierenden Vene sowie unverändertem venösen Infarkt.

zu konstanten Erweiterung nun Kaliberunregelmäßigkeiten auf (Abb.1). Weder ein Angiomnidus noch eine vorzeitige venöse Drainage war auszumachen. Unter Carbamazepin war der Patient seit einem Jahr anfallsfrei.

\section{Diskussion}

AVM sind seltene kongenitale Gefäßmissbildungen, die häufig zwischen dem 20 . und 40. Lebensjahr symptomatisch wer- 
den. Die Blutung ist mit 50\% häufigstes Initialsymptom und kann bei tiefen thalamischen AVM sogar in bis zu $90 \%$ auftreten. In etwa $25 \%$ der Patienten ist initial ein Krampfanfall wegweisend für die weitere Abklärung. Seltener sind Kopfschmerzen und sehr selten treten AVM bei Abklärung anderer Fragestellungen zutage. Spontanverschlüsse von AVM sind selten und werden mit einer Häufigkeit von 0,5\% (Lee SK et al.; Neuroradiology 2002; 44: 11; 6 von 700 Patienten mit AVM) bzw. 1,3\% (Patel MC et al.; AJNR 2001; 22: 531: 28 von 2162 Patienten) angegeben. Insgesamt sind in der Literatur 88 Fälle beschrieben, wobei bei den meisten Spontanverschlüssen intrakranielle Blutungen, partielle Embolisationen oder neurochirurgische Eingriffe vorausgingen. Von den 88 Fällen verbleiben zusammen mit unserem Fall 17 echte Spontanverschlüsse ohne bekannte vorangegangene Blutung oder Operation, die lediglich mit Kopfschmerzen oder Krampfanfällen verliefen oder asymptomatisch waren. Die bislang umfangreichste Literaturzusammenstellung von 8 Patienten mit einem eigenen Fall findet sich in der Publikation von Krapf et al. (AJNR 2001; 22:1556).

Die zugrunde liegenden pathophysiologischen Mechanismen für Spontanverschlüsse sind weitestgehend unklar. Bei Betrachtung der Lokalisation fällt auf, dass am häufigsten der Parietallappen Ort eines Spontanverschlusses ist. Aber auch Spontanverschlüsse tiefer, sogar beidseitiger thalamischer Angiome sind beschrieben (Reul et al.; Klinische Neuroradiologie 1995; 5: 46). Prädisponierende Faktoren der Angiomarchitektur scheinen wenige Feeder, kleiner Nidus und eine geringe Anzahl drainierender Venen zu sein. Diskutiert wird eine prämature Atherosklerose oder eine durch hohen Fluss induzierte Angiopathie mit Endothelläsionen, die zu überschießenden Reaktionen mit Reendothelialisierung und letztlich Verschluss der angiomversorgenden Feeder führt. Gerinnungsstörungen oder thromboembolische Embolien kardial oder aus flussassoziierten Aneurysmen sollen ebenfalls für einen Verschluss des arteriellen Schenkels des Angioms in Frage kommen. In unserem Fall mit Nachweis eines flussassoziierten Aneurysmas des proximalen parietalen Mediaastes scheint dies eher nicht zuzutreffen, da beide versorgende Feeder bis an den ehemaligen Nidus heranreichten und die frische Thrombose überwiegend in den drainierenden Venen nachzuweisen war.

Bei durch parenchymale Blutungen verursachten Spontanverschlüssen wird der raumfordernde Effekt mit Kompression des Nidus und der drainierenden Venen als Ursache diskutiert. Subarachnoidale Blutungen führen über Gefäßspasmen der angiomversorgenden Feeder möglicherweise zu Flussveränderungen, die eine Thrombose des Nidus begünstigen.
Für Spontanverschlüsse ohne begleitende Blutung oder vorangegangene Intervention scheint wie bei unserem Patienten eine Thrombose der Drainagevene und des Nidus die wahrscheinlichste Ursache zu sein. Der begleitende venöse Infarkt wurde bei unserem Patienten auch als Ursache für die Krampfanfälle gewertet. Da venöse Infarkte nicht selten zu Parenchymblutungen führen, ist eine Abgrenzung gegenüber arteriellen Angiomblutungen bildmorphologisch häufig nicht möglich.

Abdulrauf Slet al. (Neurosurgery 1999; 44: 280) berichten über Expression von VEGF und seiner Rezeptoren nach operativer Entfernung einer 4 Wochen zuvor spontan obliterierten AVM. Dies sowie die zwei publizierten Fälle (zitiert von Lee et al.; Neuroradiology 2002: 44: 11) einer Rekanalisation nach Spontanverschluss einer AVM zeigen, dass es sich hierbei um einen komplexen dynamischen Prozess handelt und Patienten mit Spontanverschlüssen langfristig nachkontrolliert werden müssen. Ebenso sollte der Nachweis von Spontanverschlüssen im Hinblick auf ihr seltenes Vorkommen nicht Anlass sein, auf eine Therapie zu verzichten.

A. Berlis, U. Pechstein, M. Schumacher, Freiburg 